\title{
Use of wireless communication networks in digitalization of factory environments
}

\author{
Tuomo Rautava ${ }^{1}$, Jarkko Paavola ${ }^{1}$, Juhani Hallio ${ }^{1}$, Juha Kalliovaara ${ }^{1}$, and Tero Jokela ${ }^{1}$ \\ ${ }^{1}$ Turku University of Applied Sciences, Turku,Finland
}

\begin{abstract}
Wireless communications are rapidly taking an important role in factory environment. The current Wi-Fi technologies struggle to meet the requirements of industrial factories, for example with regard to latency and security. The emerging $5 G$ communication networks are the first networks expected to meet such requirements. Private networks can be owned by the factories themselves. Private networks can utilize $5 G$ technologies to tailor the networks to meet the exact demands within the factory environment and guarantee that the factories can use the whole capacity for their own use. This concurrently increases the data security as the data does not need to be deliveved over public networks. The paper describes the latest developments in $5 G$ with regard to private networks and Industry 4.0, which is the name given to the digitalization, automatization and data exchange trend currently ongoing in factory environments. In this paper we describe a private cellular network we have installed inside a factory building. This network has been trialed with wireless pyrometer measurement data transmission and environmental surveillance of a measurement laboratory. The paper analyses the suitability of private networks for these use cases and discusses in general which applications would benefit the most from private wireless networks.
\end{abstract}

Keywords: Private network, factories, 5G, digitalization. 


\section{Introduction}

Wireless communications enable remote control and remote surveillance of factory environments. The currently widely used Wi-Fi technologies (eg. 802.11ac) struggle to meet the requirements of the manufacturing industry. For example, in remote control, low latency and very high reliability are required. These are not fulfilled with the shared ISM frequency bands used by Wi-Fi (2.4 GHz and $5 \mathrm{GHz}$ ). These frequencies may have other transmissions, which can lead to fluctuations in the latency and the bit rate of the Wi-Fi data transmission.

The emerging $5 \mathrm{G}$ communication networks are the first networks expected to meet such requirements. 5G technology and private networks can guarantee the desired quality of service. 5G can also create network slices optimized for different applications within the same physical network.

Turku University of Applied Sciences has examined transfer of welding data using the 5G networks and built a wireless data transmission system for temperature data of a welding robot. A 5G network can be used to transfer the temperature monitoring data during welding to a product life cycle management (PLM) system.

This research has been conducted in DigRob project, which is funded by BusinessFinland and aims to develop robotic welding to be profitable also in single-piece production.

\section{5G in factory enviroment}

The fifth-generation cellular network $(5 \mathrm{G})$ has been designed in many ways to be more versatile than its predecessors (Andrews et al 2014). With 5G, mobile network throughput can be increased to tens of gigabits per second. In addition to high throughput, $5 \mathrm{G}$ also delivers very small latency $(<1 \mathrm{~ms})$, which in turn is an enabler for applications that require real-time feedback. In addition to these enhancements, $5 \mathrm{G}$ enables the use network slicing. Network slicing allows network operators to create virtual network slices to serve, for example, various industries, self-driving cars, and basic users using the same physical network infrastructure. Network slices can be used to provide different user groups with customized service quality. For example, for connected cars, low latency is more important than a extremely high bit rate, while for a basic user a high bit rate may be more important for video viewing. The first $5 \mathrm{G}$ networks are currently being deployed and the first $5 \mathrm{G}$ terminals are entering the market.

Company-owned $4 \mathrm{G}$ or $5 \mathrm{G}$ private networks allow the company to build a network to meet its own specific needs (Matinmikko-Blue \& Latva-Aho, 2017). Thus, the network can be made very secure, necessary coverage of the network can be built, and the entire bandwidth is in the company's own use. Network computing capacity can be enhanced and end-to-end latency decreased by using local servers instead of cloud servers on the Internet. 
Private networks are built for example to factory and warehouse areas where commercial networks may be inadequate to meet the company's needs. Global Markets Insights forecasts that the global market for private mobile networks will grow to \$ 11 billion by 2024 (Global Markets Insights, 2018). Private networks are not a replacement for commercial cellular networks, but rather complementary networks for use cases where the speed or security of commercial networks is insufficient. In Finland, private networks have been built for example to Helsinki-Vantaa Airport (Nokia, 2017) and for the crane manufacturer Konecranes Hyvinkää campus (Konecranes, 2018).

The new era of automation and data transfer in the manufacturing industry is called Industry 4.0. It focuses for example on the integration and mutual exchange of information between different devices and systems, the Internet of Things (IoT) and sensing, the remote control of machines, the use of augmented reality in factory environments and other completely new services enabled by technical development. Wireless networks and especially private networks play a key role in the digitalization of industrial processes, where Finland strives to gain a significant competitive advantage by being at the forefront of development.

\section{Measurements in TUAS \#factory}

An assessment of spectrum usability in a factory environment was carried out at the TUAS \#factory at Machine Technology Center Turku by measuring the utilization rate of radio frequencies with a recording spectral analyzer. Using the spectrum analyzer, the occupancy value $(0-100 \%)$ was calculated for all the measured frequencies at $10 \mathrm{kHz}$ resolution. First, the $700 \mathrm{MHz}$ and $3.5 \mathrm{GHz}$ frequency bands were measured as they are the first bands to be used for $5 \mathrm{G}$. These frequencies have already been nationally auctioned to operators in Finland, but their use for example in the factory environments is possible through independent agreements with the operators. Figure 1 shows the spectrum analyzer hardware. The measurements concluded that no interference or other transmissions that would prevent the use of frequencies in either frequency band inside the TUAS \#factory were found. 


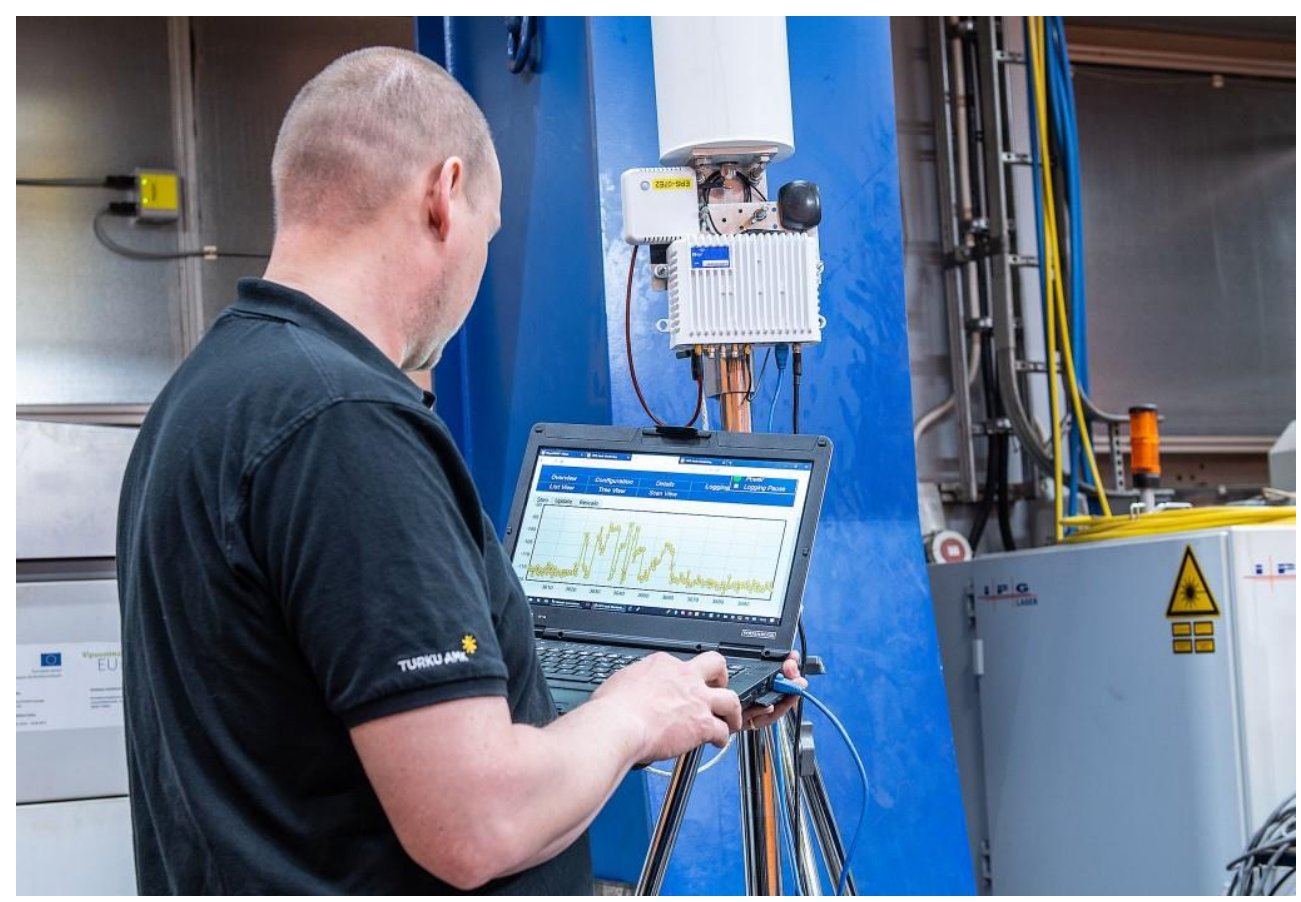

Figure 1. The CRFS RFeye spectrum measurement equipment.

After assessing spectrum availability, a private network operating on $3.5 \mathrm{GHz}$ frequency was installed inside the TUAS \#factory environment and network coverage measurements were conducted. Sufficient coverage and capacity for this environment were achieved with two low power base stations.

\subsection{Pyrometer system}

The cooling time of the weld from $800^{\circ} \mathrm{C}$ to $500^{\circ} \mathrm{C}$ is very important in determining the welding parameters applied during welding of fine-grain structural steels. This time is described by Delta T8/5 parameter. This parameter refers to the time in seconds when the temperature of the steel joint is in the range of $800^{\circ} \mathrm{C}$ to $500^{\circ} \mathrm{C}$ after welding. Stronger steels and steels with better fracture strength need to be welded more precisely in terms of the cooling time so that the properties of the base material are sufficient also after the welding. Steel manufacturers generally give their recommendation for the Delta T8/5 parameter. The cooling time can be determined by the geometry of the body, the welding parameters, or it can be measured for example by a pyrometer. 


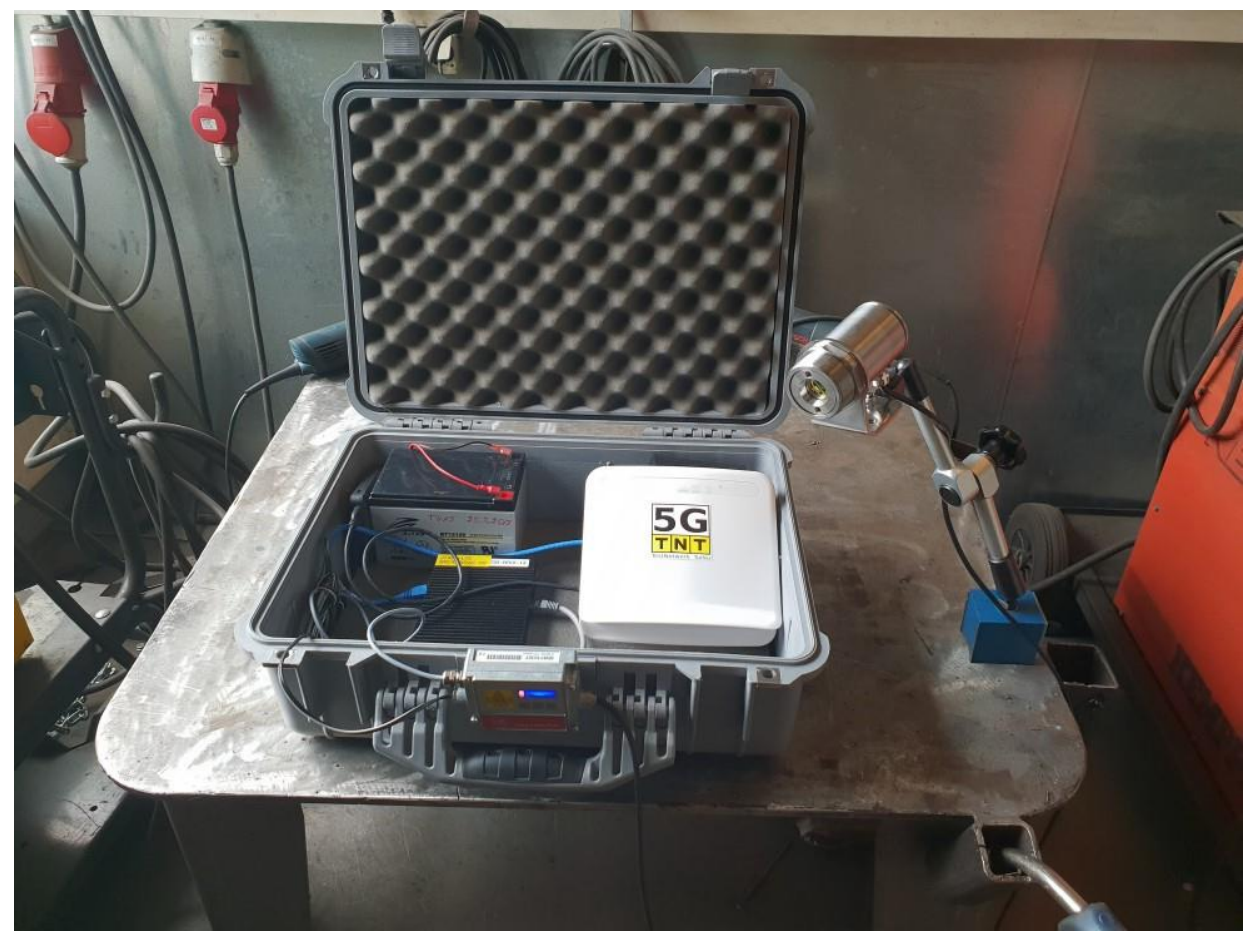

Figure 2. Wireless pyrometer setup.

In this paper, a wireless communication system was built into a commercially available pyrometer. The system is based on a cellular network technology. The built portable installation with its battery installed inside a case is shown in Figure 2. The pyrometer is installed into the metallic arm on the right side of the case. This allows measuring the cooling time of the weld at critical locations with easily portable equipment. The measured temperature data is stored locally in the memory associated with the sensor, from which it is wirelessly transferred into the cloud. The $3.5 \mathrm{GHz}$ private network installed into the factory was successfully used for data transfer. The T8/5 value is calculated from the data at the visualization and reporting stage and stored with a time stamp. The goal is to store the T8/5 value of each piece later into the PLM system. Figure 3 shows the arm and the pyrometer system installed to monitor a welding robot. 


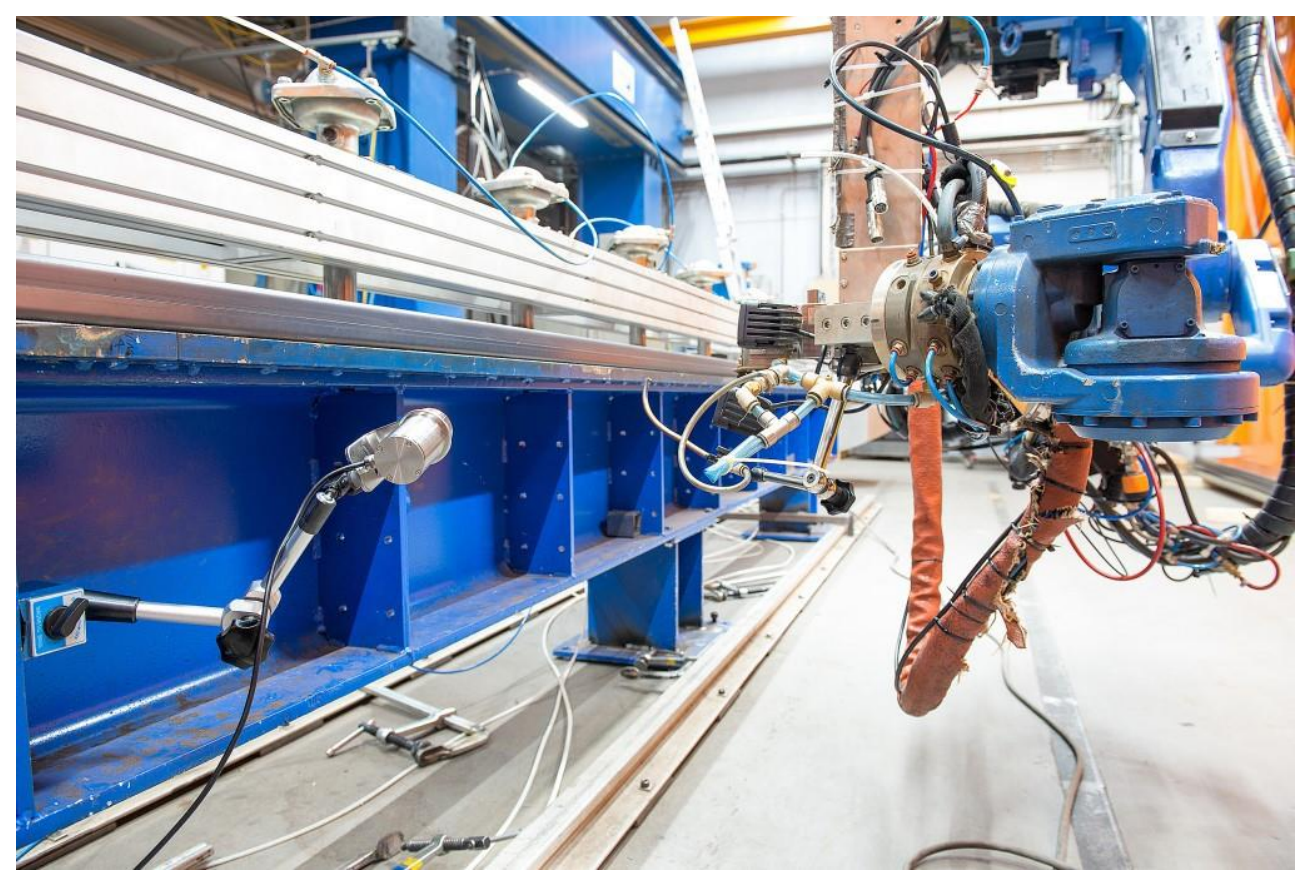

Figure 3. Pyrometer installed to the welding station.

\subsection{Measurement room sensoring system}

The conditions must remain standardized at the measurement and calibration laboratory, where the coordinate measuring machine is located. For this purpose, a wireless sensor was installed to measure the conditions inside the measurement and calibration laboratory at the Machine Technology Center. The sensor was used to measure the room temperature, humidity, lightness, and motion in the room. The sensor works wirelessly and has a battery which lasts for more than a year.

The data generated by the sensor can be visualized for remote monitoring in the same cloudbased service as with the pyrometer. If the values measured by the sensor differ from the set limit values, an alarm is given. The installed wireless system does not replace the standardized monitoring system, but allows remote monitoring of the measurement and calibration laboratory.

\section{Conclusions}

In this paper, we have taken a step towards the future factory by providing added value through a wireless private cellular network. The wireless communication system developed 
for the pyrometer makes the equipment portable, making it easy to move the equipment to a desired location, for example when welding large pieces. In turn, remote sensing of the measurement and calibration laboratory is facilitated by wireless sensors. In the future, as mobile robots, collaborative robots and remote control become more widespread, the importance of wireless communications will largely increase in factory environments.

\section{References}

Andrews, J. G. et al (2014), What Will 5G Be?, in IEEE Journal on Selected Areas in Communications, vol.32, no.6, pp.1065-1082, June 2014, doi:

10.1109/JSAC.2014.2328098

Global Markets Insights (2018), Industry Analysis Report: Private LTE Market Size By Component, Device, Service, Application and Regional Outlook, Growth Potential, Competitive Market Share \& Forecast, 2018 - 2024, November 2018.

Konecranes (2018), Konecranes, Nokia and Ukkoverkot to cooperate - smart cranes depart on the $5 G$ journey, January 2018. Available online:

https://www.konecranes.com/press/releases/2018/konecranes-nokia-and-ukkoverkot-tocooperate-smart-cranes-depart-on-the-5g-journey, Accessed June $24^{\text {th }} 2019$.

Matinmikko-Blue, M. and Latva-Aho, M. (2017), Micro operators accelerating 5G deployment, 2017 IEEE International Conference on Industrial and Information Systems (ICIIS), Peradeniya, pp.1-5. doi: 10.1109/ICIINFS.2017.8300396

Nokia (2017), Nokia to modernize private nationwide LTE network for Finland's Ukkoverkot, supporting industrial and public safety services, available online: https://www.nokia.com/about-us/news/releases/2017/09/19/nokia-to-modernize-privatenationwide-lte-network-for-finlands-ukkoverkot-supporting-industrial-and-public-safetyservices/, September 2017. Accessed June $24^{\text {th }} 2019$. 